EPJ Web of Conferences 62, 06003 (2013)

DOI: $10.1051 /$ epjconf/20136206003

(C) Owned by the authors, published by EDP Sciences, 2013

\title{
Advanced model for the prediction of the neutron-rich fission product yields
}

\author{
V. A. Rubchenya ${ }^{1,2, a}$, D. Gorelov ${ }^{1}$, A. Jokinen ${ }^{1}$, H. Penttilä ${ }^{1}$, and J. Äystö ${ }^{1}$ \\ ${ }^{1}$ Department of Physics, PO Box 35 (YFL), 40014 University of Jyväskylä, Finland \\ ${ }^{2}$ Faculty of Physics, St. Petersburg State University, St. Petersburg 198504, Russia
}

\begin{abstract}
The consistent models for the description of the independent fission product formation cross sections in the spontaneous fission and in the neutron and proton induced fission at the energies up to $100 \mathrm{MeV}$ is developed. This model is a combination of new version of the two-component exciton model and a time-dependent statistical model for fusion-fission process with inclusion of dynamical effects for accurate calculations of nucleon composition and excitation energy of the fissioning nucleus at the scission point. For each member of the compound nucleus ensemble at the scission point, the primary fission fragment characteristics: kinetic and excitation energies and their yields are calculated using the scission-point fission model with inclusion of the nuclear shell and pairing effects, and multimodal approach. The charge distribution of the primary fragment isobaric chains was considered as a result of the frozen quantal fluctuations of the isovector nuclear matter density at the scission point with the finite neck radius. Model parameters were obtained from the comparison of the predicted independent product fission yields with the experimental results and with the neutron-rich fission product data measured with a Penning trap at the Accelerator Laboratory of the University of Jyväskylä (JYFLTRAP).
\end{abstract}

\section{Introduction}

Nuclear fission is a promising source for discovering, producing, and investigating exotic nuclei with high neutron excess. Precise measurements of the fission product distributions deliver valuable information on the formation of compound fissioning nucleus and on the dynamics of the large-scale collective motion of nuclear matter. Comparative investigations of neutron-rich fission product yields in fission of heavy nuclei induced by deuterons, protons, neutrons and photons in wide range of energies are important for the development of radioactive beam facilities.

Independent fission yield measurements for proton-induced fission of ${ }^{232} \mathrm{Th}$ and ${ }^{238} \mathrm{U}$ were carried out in the Accelerator Laboratory of the University of Jyväskylä, Finland using the double Penning trap (JYFLTRAP) as a precision mass filter, which allows us to identify each nuclide on its mass [1].

Ions produced in a nuclear fission are stopped by the noble buffer gas. Due to high ionization potential of the buffer gas, considerable part of ions acquires single positive charge state $(\mathrm{q}=+1)$.

\footnotetext{
ae-mail: valery.v.rubchenya@jyu.fi
}

This is an Open Access article distributed under the terms of the Creative Commons Attribution License 2.0, which permits unrestricted use, distribution, and reproduction in any medium, provided the original work is properly cited. 
Then ions are extracted from the ion guide and accelerated up to $30 \mathrm{q} \mathrm{keV}$. The continuous beam is separated by the dipole magnet with the resolving power around 300. As result the isobaric chain with certain mass number is selected.

After the mass separation, ions are injected into the radio-frequency quadrupole [2], which cools down them and forms a shot bunch. Then bunches are delivered to the JYFLTRAP, namely, two cylindrical Penning traps placed in the $7 \mathrm{~T}$ uniform magnetic field. When ions are inside the trap, the following excitation scheme is applied. Full cycle starts from dipole excitation. Due to this all ions independently from their masses are moved to a large radius. After that applying quadrupole excitation with frequency close to a cyclotron frequency, only ions with certain mass are focused in the center of the trap. Focused ions are released from the trap through $2 \mathrm{~mm}$ diaphragm and registered by micro channel plate (MCP) detector. Thereby the mass spectrum is generated by counting how many ions were detected for each quadruple excitation frequency. Usual mass resolving power of this method is around $10^{5}$. Comparing the number of ions in the peak related to certain isotope, it is possible to obtain the isotopic yield distributions. Detailed description of the trap performance is given in the article [3] and the method of fission yield measurement is described in [1].

To analyze experimental results, a new version of the theoretical model for calculation of the spontaneous and light particle induced fission characteristics has been developed [4]. This model is a combination of new version of the two-component exciton model [5] and a time-dependent statistical model for fusion-fission process with inclusion of dynamical effects [6] for accurate calculations of nucleon composition and excitation energy of the fissioning nucleus at the scission point. The dynamical approach for isobaric width and calculating the parameters of charge polarization at scission point was used.

A description of model for calculations of fission product cross sections in the spontaneous fission, thermal neutron induced fission, and the fission of heavy nuclei induced by light particles with energies up to $100 \mathrm{MeV}$ is presented. The comparison between the theoretical predictions and experimental data obtained with JYFLTRAP for the $25 \mathrm{MeV}$ proton-induced fission of ${ }^{232} \mathrm{Th}$ and ${ }^{238} \mathrm{U}$ will be done.

\section{Theoretical model}

The theoretical model for the description of the independent fission product formation cross sections considers three main fission reaction stages: (i) the reaction mechanism of the formation of the mass, charge, and excitation energy distributions of compound nuclei ensemble; (ii) the fission process itself and the primary fission fragment formation and (iii) the deexcitation process of the heated primary fragments. In the case of spontaneous fission and low-energy neutron- and gamma- induced fission, there is only one member of the compound nuclei ensemble.

At the nucleon bombarding energy above $\sim 10 \mathrm{MeV}$, the fast pre-equilibrium emission mechanism should also be taken into account. The pre-equilibrium particle emission process is described in the frame of the two-component exciton model using Monte Carlo simulation code, which allows one to incorporate the time duration criterion for the pre-compound stage of the reaction [5]. It is supposed that duration of the pre-equilibrium process is two orders of magnitude shorter than the average statistical decay time of the initial composite nucleus. Therefore formation and de-excitation stages of the compound nuclei are considered as decoupled.

The emission of the light particles and $\gamma$ rays is assumed to start with full statistical decay width just after the end of the pre-equilibrium stage. The pre-scission neutrons and other light particles are emitted near equilibrium deformation of the compound nucleus and at descent from saddle to scission point. The fission width and the descend time is strongly influenced by nuclear friction [7].

The Monte-Carlo simulation method was used to calculate a pre-scission neutron multiplicity and spectra using the compound nuclei parameters formed after the pre-equilibrium neutron and proton emission. The time-dependent statistical approach was applied for description of particle emission 
during the fission process up to scission point to take into consideration fission the delay time and finite descent time from saddle to scission [6]. After the pre-scission particle emission, the compound nuclei arrive at the scission point with some distributions $W_{s c}\left(E_{s c}^{*}, A_{s c}, Z_{s c}, J_{s c}\right)$ over excitation energy $E_{s c}^{*}$, mass and charge numbers $\left(A_{s c}, Z_{s c}\right)$, as well as spins $J_{s c}$.

For each member of this ensemble the primary fission fragment mass, charge and excitation energy distributions are calculated. The independent fission product formation cross sections are calculated by summing over all compound nuclei and their excitation energy at the scission point

$$
\sigma_{\text {ind }}(A, Z)=\sum_{A_{s c}, Z_{s c}} \int d \sigma_{C N}^{s c}\left(A_{s c}, Z_{s c}, E_{s c}^{*}\right) / d E_{s c}^{*} \cdot Y_{\text {ind }}\left(A, Z, A_{s c}, Z_{s c}, E_{s c}^{*}\right) d E_{s c}^{*},
$$

where $\sigma_{C N}^{s c}$ is a formation cross section of given compound nucleus at the scission point. The fission product independent yields are formed in the process of neutron evaporation from excited primary fragments and calculated according to equation

$$
Y_{\text {ind }}(A, Z)=\sum_{n} Y_{\text {pre }}(A+n) P_{Z}(A+n) P_{n}(A+n, Z) .
$$

Here $Y_{\text {pre }}$ is the primary isobaric chain yield, $P_{Z}$ is the charge distribution of the primary isobaric chain, and $P_{n}(A+n, Z)$ is the n-neutron emission probability from primary fission fragment $(Z, A+n)$. The charge distribution of the primary isobaric chain is presented in the factorized form

$$
P_{Z}(A)=\widetilde{P_{Z}}(A) F_{Z}^{o e}(A),
$$

where $\widetilde{P_{Z}}(A)$ is a smoothed distribution and $F_{Z}^{o e}$ describes odd-even staggering in the charge distribution. The smoothed distribution is usually approximated by a Gaussian function

$$
\widetilde{P_{Z}}(A)=\frac{1}{\sigma_{Z}(A) \sqrt{2 \pi}} \exp \left[-\frac{(Z-\bar{Z}(A))^{2}}{2 \sigma_{Z}^{2}(A)}\right], \quad \bar{Z}(A)=A \frac{Z_{S C}}{A_{S C}}+\delta \bar{Z}(A) .
$$

Here charge polarization $\delta \bar{Z}$ is determined by the global liquid drop properties of the potential energy surface near the scission point and influenced by nuclear shell and pairing effects. To calculate $\sigma_{Z}(A)$ we shall consider the isobaric charge width as a result of a charge frozen quantal fluctuation at scission point. [8]. A fissioning nucleus at the scission point is described by two slightly overlapping fragments. The radius of the aperture $r_{\text {neck }}$ (neck radius) through which the two fragments may exchange nucleons is about $2 \mathrm{fm}$. It is assumed that the isovector nuclear density degree of freedom is much faster than the deformation and mass ones. Therefore it is possible to fix fragment masses and study the variation of the fragment charge $Z$ alone.

For small oscillations, the charge collective motion is harmonic in the smoothed potential

$$
\tilde{V}(Z)=V(\bar{Z})+\frac{1}{2} C_{Z Z}(Z-\bar{Z})^{2} .
$$

The stiffness parameter $C_{Z Z}$ and average charge $\bar{Z}$ for given mass fragments division are calculated in the framework of the scission point fission model. The scission point configuration is approximated by two nascent deformed fragments. Potential energy of the scission configuration consists of the interaction energy between fragments and their deformation energies. Deformation energy has been calculated using the Strutinsky macroscopic-microscopic method [9]. Nuclear shape was described in the lemniscates coordinate system where the Cassini ovals are used as basic figures [10]. The single particle spectra have been calculated in the axially deformed Woods-Saxon potential using universal nuclear potential parameters proposed in Ref. [11].

The potential energy at scission point was minimized by variation of deformation parameters of both fragments, and for a given compound nucleus the two-dimensional function of $V_{\min }(A, Z)$ was computed. At fixed fragment mass division, this potential energy was approximated by the parabolic dependence and parameters $\bar{Z}(A), C_{Z Z}(A)$ were calculated. The odd-even staggering and nuclear shell effect manifest in the dependence of $\bar{Z}(A)$ and $C_{Z Z}(A)$ relative to the values predicted by the liquid 
drop model. The standard deviation of the smoothed charge distribution in equation (4) is defined by the formula

$$
\sigma_{Z}(A)=\frac{1}{2} \frac{\hbar}{\sqrt{M_{Z Z} C_{Z Z}}}, \quad M_{Z Z}=\frac{16}{9} r_{0}^{3} m \frac{A_{C N}}{Z_{C N} N_{C N}} \frac{l_{\text {neck }}+2 r_{\text {neck }}}{r_{\text {neck }}^{2}},
$$

where the inertia parameter the formula derived in Ref. [12] was used, and $r_{0}$ is a nuclear radius parameter, $m$ is a nucleon mass, $l_{\text {neck }}$ is a neck length $\left(l_{\text {neck }}=2-4 f m\right)$, and $r_{\text {neck }}$ is a neck radius $\left(r_{\text {neck }}=2 \mathrm{fm}\right)$. The odd-even correction function $F_{Z}^{o e}$ in the equation (4) was approximated by function

$$
F_{Z}^{o e} \propto \exp \left(\Pi_{Z}^{H}+\Pi_{Z}^{L}\right) \delta_{Z}\left(A_{C N}, Z_{C N}, E_{C N}\right),
$$

where the proton number parities in light $\pi_{Z}^{L}$ and heavy $\pi_{Z}^{H}$ fragments are equal to 1 (even $Z$ ) or -1 (odd $Z$ ). The proton odd-even differences parameter $\delta_{Z}\left(A_{C N}, Z_{C N}, E_{C N}\right)$ is parameterized in accordance with experimental data [13].

The pre-neutron emission isobaric chain yield is also presented in the factorized form

$$
Y_{\text {pre }}(A)=\tilde{Y}_{\text {pre }}(A) F_{A}^{o e} .
$$

The odd-even correction for the primary fragment mass distribution includes the proton and neutron corrections which are defined by the equation (7) with corresponding parameters for neutrons [4].

Parameterization of smoothed mass distribution is based on the multimodal nature of nuclear fission [14], depicting the influence of nuclear-shell structure on the potential-energy surface (PES) of fissioning nucleus. To proceed into very asymmetric fragment mass region, two additional superasymmetric fission modes were added. The five fission modes: symmetric $(S Y)$, standard-I (SI), standard-II (SII), superasymmetric-I ( $S A I)$ and superasymmetric-II (SAII) were taken into consideration to approximate the smoothed primary mass distribution [14]:

$$
\tilde{Y}_{\text {pre }}(A)=C_{S Y} Y_{S Y}(A)+C_{S I} Y_{S I}(A)+C_{S I I} Y_{S I I}(A)+C_{S A I} Y_{S A I}(A)+C_{S A I I} Y_{S A I I}(A) .
$$

Symmetric component $Y_{S Y}$ corresponds to a global liquid drop valley on PES in the multidimensional deformation parameter space. Nuclear shells modulate potential surface creating additional valleys which disappear at high compound nucleus excitation energy. The component $Y_{S I}$ is connected with $Z=$ 50 and $N=82$ nuclear shells in heavy fragments $\left({ }^{132} \mathrm{Sn}\right.$-mode), and $Y_{S I I}$ component is influenced by the deformed nuclear shell at $N=86-90$. The superasymmetric-I mode $Y_{S A I}$ and superasymmetric-II mode $Y_{S A I I}$ are supposed to be connected with $N=50$ neutron shell, and proton shell $Z=28\left({ }^{78} \mathrm{Ni}\right.$ mode) in light fragments. The dependences of these parameters on the compound nuclei excitation energy has been parameterized using the experimental data from the proton induced fission of ${ }^{232} \mathrm{Th}$, ${ }^{238} \mathrm{U}$, and ${ }^{242} \mathrm{Pu}$ measured with HENDES setup in Jyväskylä [15-17].

The neutron multiplicity distribution for the accelerated primary fission fragments $P_{n}(A, Z)$ in the formula (2) is calculated within standard statistical model. The fragment kinetic and excitation energies were calculated in the framework of the fission scission point model. Computer code FIPRODY (FIssion PRODuct Yield) was developed to calculate the main characteristics of the spontaneous fission and light particles induced fission and photo-fission.

\section{Results and discussions}

Comparison of the measured with JYFLTRAP and calculated independent isotopic yield distributions for germanium and barium isotopic chains in the proton induced fission of ${ }^{232} \mathrm{Th},{ }^{238} \mathrm{U}$ at $E_{p}=25 \mathrm{MeV}$ are shown in the Figures 1 and 2. The experimental points were fitted with Gaussian and the Gaussian's area was normalized to the calculated total yield. The main sources of uncertainty are the intensity of the mass peak (statistical uncertainty), decay correction, unresolved isomeric states and other corrections.

The model gives also good description of the fission product yields in the spontaneous fission and the neutron-induced fission. As example, the comparison the calculated formation fission product cross 

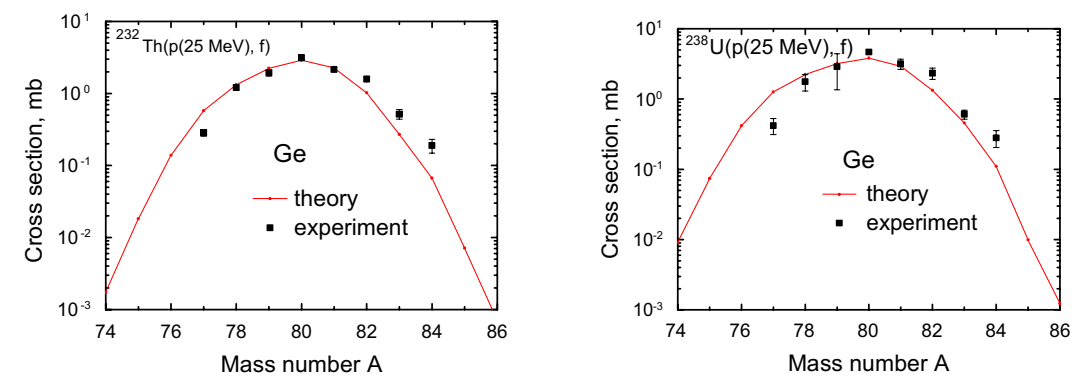

Figure 1. Comparison of the theoretical cross sections (line) and measured independent yields (points with errors) of the germanium isotopes for the proton-induced fission of ${ }^{232} \mathrm{Th}$ and ${ }^{238} \mathrm{U}$ at the $25 \mathrm{MeV}$ beam energy.
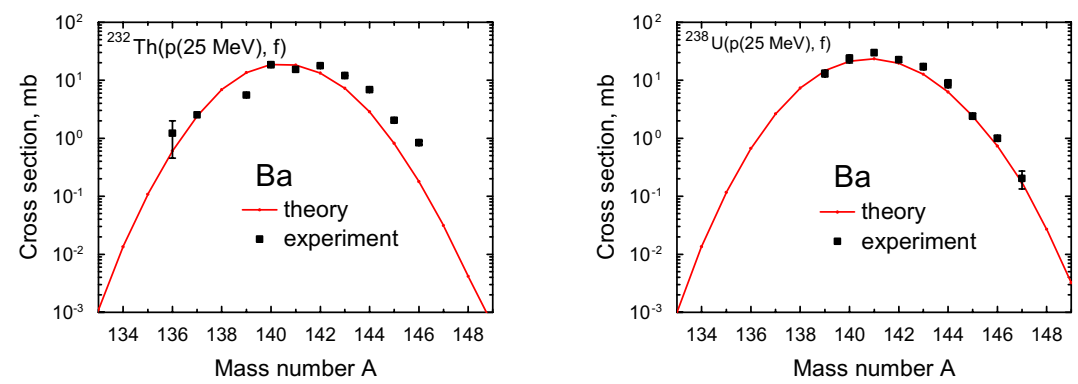

Figure 2. As in Figure 1 for the barium isotopes.

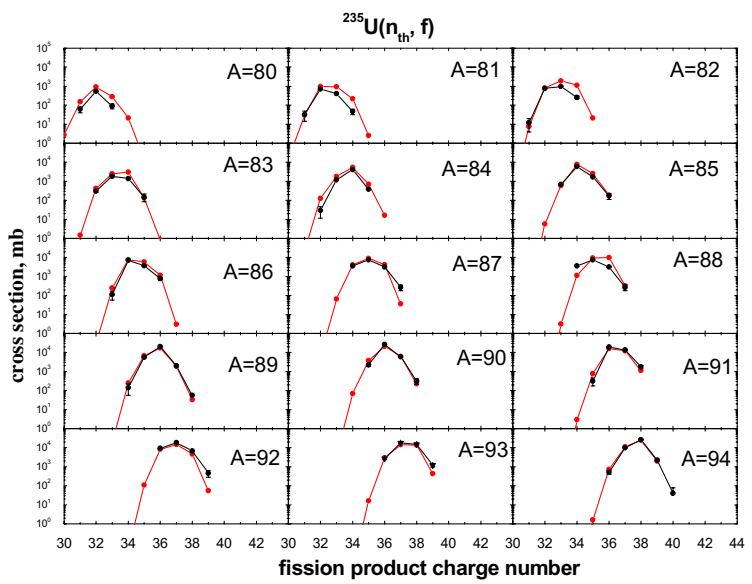

Figure 3. Theoretical formation fission product cross sections in comparison with the LOHENGRIN data for the isobaric chains $\mathrm{A}=80-94$ in the thermal neutron induced fission of ${ }^{235} \mathrm{U}[18]$.

sections for the isobaric chains $A=80-94$ in the thermal neutron-induced fission of ${ }^{235} \mathrm{U}$ with the LOHENGRIN experimental data (points with error bars) [18] is shown in the Figure 3.

The neutron-induced fission of the neutron-rich heavy elements is a suitable reaction for the production of very neutron-rich fission products. The predicted Sn product yields in the thermal neutroninduced fission of heavy nuclides ${ }^{235,241,249} \mathrm{U},{ }^{247} \mathrm{Cm}$, and ${ }^{251} \mathrm{Cf}$ are presented in the Figure 4 . The most 


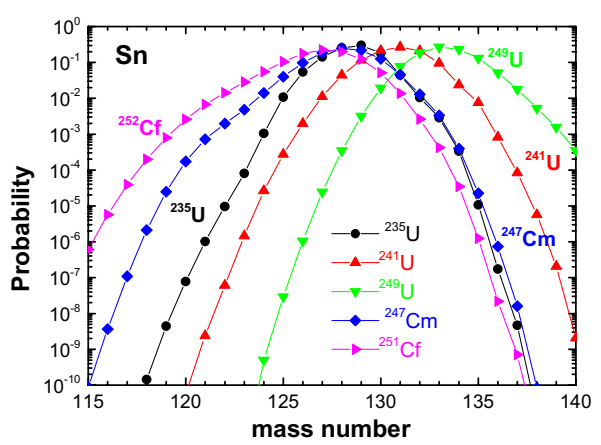

Figure 4. Calculated fission product yields of the $\mathrm{Sn}$ isotopes in the thermal neutron induced fission of ${ }^{235,241,249} \mathrm{U}$, ${ }^{247} \mathrm{Cm}$, and ${ }^{251} \mathrm{Cf}$ (distributions are normalised to 1 ).

neutron-rich heavy compound nuclei may be formed in the transfer reactions using the neutron-rich radioactive beams.

The present model and computer code provide a powerful tool for the experimental data analysis and practically important relevant nuclear data predictions. The calculation method has a special importance for searching experimental method for the extremely neutron-rich nuclide production.

This work is supported by the Academy of Finland under project No. 139382, the Finnish Center of Excellence Program 2006 - 2011 (Project No. 213503, Nuclear and Accelerator Based Physics Program at JYFL) and by European Union within EURISOL design study.

\section{References}

[1] H. Penttilä et al., Eur. Phys. J. A 44, 147 (2010)

[2] A. Nieminen et al., Nucl. Instrum. Methods Phys. Res., Sec. B 204, 563 (2003)

[3] T. Eronen et al., Eur. Phys. J. A 48, 46 (2012)

[4] V.A. Rubchenya, J. Äystö, Eur. Phys. J. A 48, 44 (2012)

[5] V.A. Rubchenya, Phys. Rev. C 75, 054610 (2007)

[6] V.A. Rubchenya et al., Phys. Rev. C 58, 1587 (1998)

[7] P. Grangé, S. Hassani, H.A. Weidenmüller, A. Gavron, J.R. Nix, A.J. Sierk, Phys. Rev. C 34, 209 (1986)

[8] H. Nifenecker, Journal de Phys.-Lett., France, 41 (1980) L47

[9] V.M. Strutinsky, Nucl. Phys. A 122, 1 (1968)

[10] V.V. Pashkevich, Nucl. Phys. A 169, 275 (1971)

[11] S. Cwiok, J. Dudek, W. Nazarewicz, J. Skalski, T. Werner, Comp. Phys. Communications 46, 379 (1987)

[12] H.E. Hernandes et al., Nucl. Phys. A 361, 483 (1981)

[13] J.P. Bocquet, R. Brissot, Nucl. Phys. A 502, 213c (1989)

[14] I. Tsekhanovich et al., Phys. Rev. C 70, 044610 (2004)

[15] V.A. Rubchenya et al., Nucl. Instrum. Methods Phys. Res., Sect. A 463, 653 (2001)

[16] V.A. Rubchenya et al., Nucl. Phys. A 734, 253 (2004)

[17] E.M. Kozulin et al., AIP Conf. Proc., vol. 853, p. 336-341, 2006

[18] W. Lang et al. Nucl. Phys. A 345, 34 (1980) 